\title{
Pain Intensity and Function Improvement in Shoulder Impingement Syndrome with Kinesio Taping without Specific Technique
}

\author{
Andhitya Dwi Ananda, Tirza Z. Tamin, I Nyoman Murdana1 \\ Department of Medical Rehabilitation Dr. Cipto Mangunkusumo National Hospital, Faculty of \\ Medicine, University of Indonesia, Jakarta-Indonesia.
}

\begin{abstract}
Background: Shoulder Impingement Syndrome (SIS) can occur in three age groups according to the stage of its disease. The First stage of SIS mostly occurs in below 25 years old, while the second stage occurs in 25-40 years old and third stage occur above 40 years old. The effectiveness of Kinesio Taping in SIS using Kaze's technique or modification of the technique has been proven to have positive benefits according to the previous studies. However, the Kinesio taping have some challenges, like it has to replaced after some days application, it cannot apply by the patient, and it has some contraindications like an infection in the affected area, deep vein thrombosis, allergy to the tape, wound, and some other conditions

Methods: This study used pre and post-trial without control on subjects with SIS. The "sham" Kinesio Taping application consists of three I-strips that applied with no tension for one week, with reapplication in three days. The outcomes were Pain intensity that was measured by Visual Analog Scale (VAS), flexion, abduction, and scaption range of motion, and quality of life that scored by the Quick Disability of Arm, Shoulder, and Hand (DASH) measurement tool.
\end{abstract}

Result: Study subjects were 16 people with mean age is 59-year-old. There was a significant improvement in pain intensity, both with movement and nocturnal pain $(\mathrm{p}<0.05)$. The range of motion also showed significant improvement in all three measurements: pain-free shoulder abduction, flexion, and scaption compared to the pre-test values $(\mathrm{p}<0.05)$. Quick DASH score also showed significant improvement compared to the pre-test values $(\mathrm{p}<0.05)$.

Conclusion: Kinesio Taping may represent a useful adjunct therapy in the management of SIS. Results of the present study may challenge the suggested mechanisms for the effectiveness of Kinesio Taping.

Keywords: Kinesio taping, Kaze technique, Shoulder impingement syndrome(SIS), allergy, deep vein thrombosis, Visual Analog Scale (VAS), Quick Disability of Arm, Shoulder, and Hand (DASH)

\section{Correspondence Detail:}

Andhitya Dwi Ananda,

Email : andhityadwiananda@gmail.com 


\section{INTRODUCTION}

Shoulder pain is one of the highest prevalence of musculoskeletal complaints that make patients seek medical help. Several studies suggest that the current prevalence of shoulder pain complaints ranges from $7 \%-36 \%$ of the population. Shoulder impingement syndrome (SIS) is the most common cause of shoulder pain complaints in patients coming to Orthopedic or Physical and Medical Rehabilitation outpatient clinics. It is a result of compression of the supraspinatus tendon, subacromial bursa, and other structures as they pass through the space between the acromion and the humeral head (Greenberg, 1998). Data from Cipto Mangunkusumo National General Hospital in 2014 showed 245 6,144 patient cases with SIS. ${ }^{1-4}$

Patients with SIS will experience functional limitation, particularly difficulty in performing the usual work and lifting weights above the head. Prolonged SIS will result in significant functional disability and reduction of quality of life. Early management in SIS is given nonoperatively, thus putting rehabilitation as the first line of therapy. ${ }^{5}$

There are two basic application directions for treatment of muscles. For acutely over-used or stretched muscles, the tape is applied from insertion to origin to inhibit muscle function. Insertion to origin application tape stretch/tension is very light or light, $15-25 \%$ of available tension. For chronically weak muscles or where increased contraction desired, the tape is applied from origin to insertion to facilitate muscle function. Origin to insertion application tension is light to moderate, with $25-50 \%$ tension. The aim of Kinesio Taping application in SIS is muscle and fascia correction that leads to improvement in muscle coordination as well as improvement in centering the head of the humerus. ${ }^{6}$

Kinesio Taping is presumed to work through hypothetical physiological mechanisms. The most accepted hypothesis to date is the Gate Control theory. The theory discovered by Melzack and Wall in 1965 is responsible for relieving pain in specific body locations when intensive tactile stimulation applied at the same site. The mechanical stimuli produced by Kinesio Taping play through the fast conductive nerve fibers $(A \beta)$, which are synthesized with interneuron inhibition when it reaches the Roland gelatinous substance in the posterior horn of the spinal cord. The neural fibers $A \beta$, which is a sensory neuron for touch, has a larger diameter and faster conduction velocity than the nerve fibers of pain, including neural fibers A $\delta$ and nerve fibers $C$. Transmission of pain will inhibit at the level of the spinal cord, so it does not reach the cortex. ${ }^{7}$

Application of Kinesio Taping has become more popular in recent years. Kinesio Taping considered as an option to control the movement of the scapula in patients with shoulder problems. Kinesio Taping commonly used in the rehabilitation scope as both prevention and treatment of musculoskeletal disorders. The function of Kinesio Taping is to provide support during movement. The mechanism underlying the Kinesio Taping effect is still debatable. Speculation that arises is Kinesio Taping works by inducing proprioceptive feedback or providing corrections of alignment during dynamic movements., ${ }^{2,8}$ 
The effectiveness of Kinesio Taping in SIS using Kaze's technique or modification of the technique has been proven to have positive benefits from previous studies. Nevertheless, some studies have also found a positive effect of sham kinesiotaping without specific techniques. The question then arises whether a kinesiotaping applications without specific techniques at the tender point can also provide similar benefits. 2, 9-12

This theory supported by a systematic review of Artioli et al. The stress generated by taping generating an afferent stimulus that facilitates the mechanism of inhibition of pain. Kinesio Taping provides sensorimotor feedback which only permits the movement that caused a minimal tissue irritation, resulting in lower pain. ${ }^{13}$

Kim and Lee assumed that the elasticity of Kinesio Taping could induce skin deformation that stimulates the cutaneous mechanoreceptors. According to the counterirritant theory, the mechanoreceptor afferent excitation induces the release of Enkefalin, which inhibits the internal hyperpolarization of the interneuron and the neurotransmitter involved in pain integration, i.e., substance P. The result of the process is the inhibition of nociceptive signal transmission ${ }^{14}$

Chang et al. suggest a lifting effect mechanism by Kinesio Taping that can reduce pain with increased circulation under the injury area. Increased circulation is expected to accelerate tissue repair and rotation of inflammatory substances. ${ }^{15}$

Shakeri et al. performed modified of the Kase's Kinesio Taping technique by adding a Y -strip to the lower trapezius muscle with $50 \%$ stretch to involve the scapulohumeral control. This study also compared with sham Kinesio Taping with the same method as Thelen et al. The results were significant improvements in the intensity of pain during movement, night pain, and pain-free flexion and abduction range of motion after Kinesio Taping in the intervention group. While in the sham group there was no significant difference in all aspects of the assessment, except for abduction range of motion.

Improvement in range of motion obtained in this study is assumed to be due to the improvement of pain. Besides, sensorimotor feedback provided by Kinesio Taping application provides symptom improvement and increased level of comfort, which was suspected to be the cause of the improvement in Quick Dash score.

\section{MATERIAL AND METHODS}

\section{Design}

This study used pre and post-trial without control of subjects with SIS aged 18-75 years.

\section{Participant}

The sampling technique used was consecutive sampling subjects with SIS aged 18-75 years. All of the 
patients presenting to the Physical Medicine and Rehabilitation outpatient clinic in Cipto Mangunkusumo National General Hospital. The subjects have positive on two or more shoulder impingement screenings, i.e.: a history of pain on the anterior or lateral shoulder that weighs more than two weeks during the last six months. There was a painful arc sign at active shoulder elevation, pain in palpation of rotator cuff tendon, abduction pain shoulder with resistive isometric. Moreover, there was a positive Jobe test, Moderate pain (scale 4-7 on Visual Analog Scale), positive on one or more of the shoulder impingement-specific tests, i.e., the Neer test or the Hawkins-Kennedy test, definite signs of impingement on dynamic examination of shoulder ultrasonography considered for enrollment in this study. Exclusion criteria were: History of dislocation, fracture, or traumatic injuries within the shoulder complex and did not take medicine especially analgetic, history of shoulder surgery within the last six months, Complete rupture of rotator cuff muscles with acute inflammation, History of cervical involvement and Had a contraindication to Kinesio Taping.

\section{Outcome measure}

We enrolled 16 subjects in the study. The "sham" Kinesio Taping application in tender point area is provided for one week, with reapplication in three days. The sham Kinesio Taping consisted of three I-strips that applied with no tension. The first piece of tape placed over the acromioclavicular joint, the second one on the distal deltoid, and the third one applied on the lower trapezius muscle, all without any tension (Figure 1). The sites were selected because they are the most common locations of perceived pain by patients with rotator cuff tendonitis or impingement.

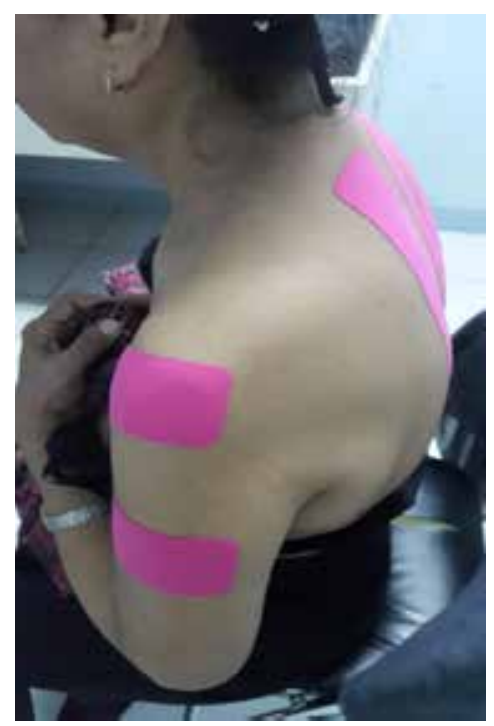

A

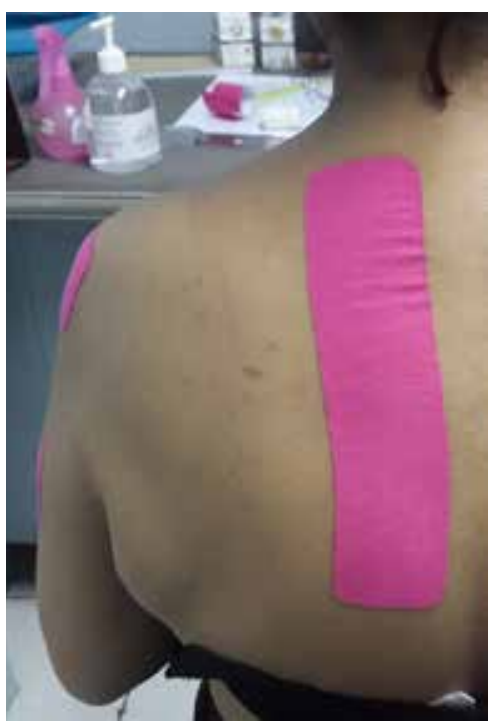

B

Figure 1. The Application of Sham Kinesio Taping. Lateral view (A) and posterior view (B).

The outcomes were Pain intensity that was measured by Visual Analog Scale (VAS), Range of Motion that was measured by Goniometer, and quality of life that scored with Quick DASH tool. Pain intensity consists of pain with movement and nocturnal pain. A range of motions that were measured were flexion, abduction, and caption. 
Quick DASH scores were used to assess physical disability and symptoms in patients with upper extremity musculoskeletal abnormalities. This score contains 11 questions with a range of 0 (not normal) to 100 (normal). All subjects provided written informed consent, evaluated for socio-demographic and initial examination.

Saphiro Wilk analysis was conducted to test the data distribution. Mann Whitney parametric test was used to was used to determine whether significant differences existed between pre and post-KinesioTaping application values. The analysis was conducted using SPSS version 17.0.

\section{RESULTS}

Baseline characteristics and initial assessment of each outcome measure of the subjects has shown in Table 2.

Table 2. Subjects Characteristics

\begin{tabular}{lr}
\hline \multicolumn{1}{c}{ Characteristics } & \multicolumn{1}{c}{$\begin{array}{c}\text { Sham } \\
\mathbf{n = 1 6}\end{array}$} \\
\cline { 2 - 2 } & $\mathbf{n}(\mathbf{\%})$ \\
\hline Mean Age (year) & $59.69 \pm 9.55$ \\
Sex & \\
$\quad$ Man & $8(50 \%)$ \\
$\quad$ Woman & $8(50 \%)$ \\
Dominant Hand & \\
$\quad$ Right & $16(100)$ \\
$\quad$ Left & $0(0)$ \\
Analgetic Consumption & \\
$\quad$ None & $12(75)$ \\
$\leq 3$ & $2(12.5)$ \\
$>3$ & $2(12.5)$ \\
\hline
\end{tabular}

Study subjects were 16 people with mean age is 59-year-old. Male and female were equal. Nutritional status is dominated by obesity, and the education of subjects are dominated by advanced level.

Most of the study subjects were housewives, the rest as retirees, employees, civil servants, and teachers. The pain in the Kinesio Taping group was dominated by the right side. There was one subject (6.3\%) of the Kinesio Taping group and two subjects $(12.5 \%)$ of the sham group who consumed more than three analgesics during the research process. 
The primary outcome results were the significant improvement in pain intensity, both with movement and nocturnal pain $(\mathrm{p}<0.05)$. The range of motion also showed significant improvement in all three measurements: pain-free shoulder abduction, flexion, and scaption compared to the pre-test values $(\mathrm{p}<0.05)$. Quick DASH score also showed significant improvement compared to the pre-test values $(\mathrm{p}<0.05)$. The main results showed in Table 3 .

Table 3. Outcome Results

\begin{tabular}{lrrrrr}
\hline \multicolumn{1}{c}{ Outcome } & \multicolumn{1}{c}{ Before } & $\begin{array}{c}\text { Immediately } \\
\text { after }\end{array}$ & After three days & After one week & Sig \\
\hline $\begin{array}{l}\text { Pain with } \\
\text { movement }\end{array}$ & $5.13 \pm 1.15$ & $4.62 \pm 1.54$ & $3.69 \pm 1.78$ & $3.19 \pm 1.80$ & 0.000 \\
Nocturnal pain & $5.00 \pm 1.21$ & $4.40 \pm 1.55$ & $3.56 \pm 1.82$ & $3.06 \pm 1.88$ & 0.007 \\
Flexion & $134.38 \pm 33.61$ & $141.25 \pm 31.50$ & $149.69 \pm 27.84$ & $154.06 \pm 29.28$ & 0.002 \\
Abduction & $107.50 \pm 36.50$ & $119.06 \pm 37.25$ & $124.69 \pm 34.47$ & $135.31 \pm 38.10$ & 0.000 \\
Scaption & $122.81 \pm 33.01$ & $130.94 \pm 34.31$ & $138.44 \pm 31.87$ & $147.81 \pm 30.87$ & 0.000 \\
Quick Dash & $43.66 \pm 13.80$ & - & $34.09 \pm 16.98$ & $31.44 \pm 16.59$ & 0.000 \\
\hline
\end{tabular}

\section{DISCUSSION}

The results of this study showed a significant improvement in pain with movement, nocturnal pain, and pain-free shoulder abduction, flexion and scaption ROM after sham Kinesio Taping as compared to the pre-test values.

Literature review on the use of Kinesio Taping in the management of shoulder abnormalities is still limited. Randomized controlled clinical trials from Thelen et al. investigated the application of Kinesio Taping on shoulder pain using the Kase method. The technique consisted of two pieces of Y-strip applied from the origin to the insertion of the deltoid and supraspinatus muscles, respectively, with paper off tension, and one I-strip applied from the coracoid process to the posterior deltoid with 50-75\% stretch for correction of glenohumeral mechanics. The study compares with Sham Kinesio Taping consisted of three I-strips with no tension in tender point area. The result of the study was the improvement of pain-free abduction range of motion after Kinesio Taping application. ${ }^{1}$

A randomized study from Kocyigit et al. (2016) compared Kinesio Taping application with sham in SIS. Results from 41 participants, 13 were males (32\%), and 28 were females (68\%). The mean age was $45 \pm 15$ years (range 20-65 years). We documented a significant decrease in VAS for nocturnal pain and Constant Score in both groups. The KT group showed the significant additional change in NHP pain and physical 
activity scores. In this Study subjects were 16 people with mean age is 59-year-old. There was a significant improvement in pain intensity, both with movement and nocturnal pain $(\mathrm{p}<0.05)$. The range of motion also showed significant improvement in all three measurements: pain-free shoulder abduction, flexion, and scaption compared to the pre-test values $(\mathrm{p}<0.05)$. Quick DASH score also showed significant improvement compared to the pre-test values $(\mathrm{p}<0.05)$. The sham tape used surgical hypoallergenic flexible tape with the identical method as Kinesio Taping, but no force was applied. The exciting result is that sham taping also provided a significant decrease in nocturnal pain. They explained that the long-lasting analgesic effect of sham taping might be due to the activation of the gate control theory. From another point of view, it can be proposed that the reduction in pain not be related to a taping. Regular exercising, modification of daily living activities together with spontaneous healing may alleviate pain in both groups. ${ }^{16}$

Another Randomized-controlled study from Kocyigit et al. (2015) investigated the effects Kinesio Taping versus Sham application on pain, functional performance and quality of life in patients with knee osteoarthritis. They also used identical strips with Beta fix Surgical Hypoallergenic Flexible Tape as the sham. Kinesio Taping improved Lequesne index scores, Nottingham Health Profile (NHP) pain, and physical activity scores significantly in this study. Interestingly, Sham taping also improved these scores significantly. Moreover, comparisons between the groups documented significantly higher NHP energy and NHP total scores in favor of sham taping group at the end of the treatment period. The study cannot explain the underlying mechanisms for the superiority of sham taping over Kinesio Taping. ${ }^{12}$

The present study shows improvement in the pain scale, both nocturnal pain and during movement after sham Kinesio Taping application. This is thought to be due to sensorimotor feedback effect and Lifting Effect that also occurred when Kinesio Taping is attached to the skin, even without a particular technique. Moreover, Kumbrink has explained that the adhesion of the Kinesio Taping to the skin, and the resulting mechanical displacement caused by body movement, leads to stimulation of the mechanoreceptors in the skin. Like the nociceptive afferents, these proprioceptive afferents also run to the dorsal horn and inhibit the relaying of nociception. Further studies needed to confirm this theory. ${ }^{17}$

This study is a follow-up study of a study comparing Kinesio Taping application based on Kase's modification theory performed by Shakeri et al. with Sham Kinesio Taping application performed in this study. According to Salaffi et al., Minimally Clinically Important Difference (MCID) for VAS scores on the $\backslash$ musculoskeletal pain intensity is $3.00-3.50 \mathrm{~cm}$. In the main study, the delta VAS score of movement and night before and after seven days of Kinesio Taping application was in the MCID range (3.31 \pm 1.20 and $3.50 \pm 1.21$, respectively). While in the sham group, the improvement in VAS score did not reach MCID value. ${ }^{18,19}$

The results of the present study need to be viewed in light of several limitations. This study had no control group, in which to know the effectiveness of Kinesio Taping more objectively required controls that were not given any treatment, or just given a tape other than Kinesio Taping. Thus, the author did not involve the control group because of ethical consideration. Moreover, the study was performed over a 7-day period, 
thus excluding analysis of long-term benefits. Although the present study shows the short-term relief of pain, studies utilizing a more extended period are warranted to determine if there is a continued reduction in symptoms and if taping remains valid over time. Lastly, the placebo effect as the main confounder should also be considered.

\section{CONCLUSION}

Kinesio Taping may represent a useful adjunct therapy in the management of SIS. Results of the present study may challenge the suggested mechanisms for the effectiveness of KT. Therefore, further researches are required to resolve this challenge.

\section{REFERENCES}

1. Thelen MD, Dauber JA, Stoneman PD. The Clinical Efficacy of Kinesio Tape for Shoulder Pain: A Randomized, Double-Blinded, Clinical Trial. journal of orthopaedic \& sports physical therapy. 2008;38(7):389-95.

2. Shakeri H, Keshavarz R, Arab AM, Ebrahimi I. Clinical Effectiveness of Kinesiological Taping on Pain and Pain Free Shoulder Range of Motion in Patients with Shoulder Impingement Syndrome: a Randomized, Double Blinded, Controlled Trial. The International Journal of Sports Physical Therapy. 2013;8(6):800-11.

3. Green S, Buchbinder R, Hetrick S. Physiotherapy interventions for shoulder pain. Cochrane Database Syst Rev. 2003;2.

4. Greenberg DL. Evaluation and treatment of shoulder pain. Medical Clinics of North America. 2014;3:487-504.

5. Chipchase LS, DA DAOC, Costi JJ, Krishnan J. Shoulder impingement syndrome: preoperative health status. J Shoulder Elbow Surg. 2000;9(1):12-5.

6. Kase K. Ilustrated Kinesio Taping. Tokyo: Ken Ikai; 2003.

7. Gosling AP. Mecanismos de ação e efeitos da fisioterapia no tratamento da dor. Rev Dor. 2013;13(1):6570.

8. Halseth T, McChesney JW, DeBeliso M. The effects of Kinesio taping on proprioception at the ankle. J Sports Science Med. 2004;3:1-7.

9. Jaraczewska E, Long C. Kinesio ${ }^{\circledR}$ taping in stroke: Improving functional use of the upper extremity in hemiplegia. Top Stroke Rehabil. 2006;13(3):31-42.

10. Stoller HR, The Effect of McConnell Shoulder Taping on Shoulder Muscle EMG and Shoulder Torque Productions in Subjects With Anterior Shoulder Instability. Seton Hall University Dissertations and Theses.2013

11. Kaya E, Zinnuroglu M, I.Tugcu. Kinesio taping compared to physical therapy modalities for the 
treatment of shoulder impingement syndrome. Clin Rheumatol. 2011;30:201-7.

12. Kocyigit F, et al. Kinesio taping or sham taping in knee osteoarthritis? A randomized, double-blind, sham-controlled trial. Complementary Therapies in Clinical Practice. 2015;21(4):262-7.

13. Artioli DP, Bertolini GRF. Kinesio taping: application and results on pain: systematic review. Fisioter Pesqui. 2014;21(1).

14. Kim B-J, Lee J-H. Efficacy of Kinesiology Taping for Recovery from Occupational Wrist Disorders Experienced by a Physical Therapist. J Phys Ther Sci. 2014;26(6):941-3.

15. Chang H-Y, Wang C-H, Chou K-Y, Cheng S-C. Could Forearm Kinesio Taping Improve Strength, Force Sense, and Pain in Baseball Pitchers With Medial Epicondylitis? Clin J Sport Med. 2012;22(4).

16. Kocyigit $\mathrm{F}$, et al. Kinesio taping or just taping in shoulder subacromial impingement syndrome? A randomized, double-blind, placebo-controlled trial. Physiotherapy Theory and Practice : An International Journal of Physical Therapy. 2016.

17. Kumbrink B. K-Taping: An Illustrated Guide - Basics - Techniques - Indications. 2nd ed: Springer; 2014.

18. Salaffi F, Stancati A, Silvestri CA, Ciapetti A, Wallis J. Minimal clinically important changes in chronic musculoskeletal pain intensity measured on a numerical rating scale. Eur J Pain. 2004;8(4):283-91.

19. Ananda AD. Pengaruh Kinesio Taping terhadap Intensitas Nyeri, Lingkup Gerak Sendi, dan Skala Disailitas pada Sindrom Impingement Bahu Jakarta: Faculty of Medicine University of Indonesia; 2017. 